Article

\title{
A Conceptual Framework for Implementing a WSN Based Cattle Recovery System in Case of Cattle Rustling in Kenya
}

\section{Patrick Kanyi Wamuyu}

School of Science and Technology, United States International University, 14634-00800 Nairobi, Kenya; kanyiwamuyu@yahoo.com or pwamuyu@usiu.ac.ke

Received: 26 June 2017; Accepted: 28 August 2017; Published: 31 August 2017

\begin{abstract}
This study proposes a framework for remote identification and tracking of cattle movement based on wireless sensor networks (WSN), mobile communication, and unmanned aerial vehicles (UAVs). The proposed framework can be implemented for tracking cattle movement at the village level and extended to harsh terrain when recovering stolen animals in case of cattle rustling or theft. The system works by identifying and tracking the desired animal location and sending periodic location data at regular intervals to a database as well as availing the specific animal's current location on demand through the Internet and text messages. The proposed cattle tracking and recovery system (CTRS) consists of a rumen sensor module, a WSN control unit, a Worldwide Interoperability for Microwave Access (WiMAX) gateway, WiMAX base stations, and a data center. Availing timely information about the location of the stolen animals could facilitate quick recovery of the animals while ensuring the safety of the security personnel involved in the recovery process. Speedy recovery of stolen animals also defeats the purpose of cattle rustling as the rustlers do not have the chance to use the animals for their intended purposes. The proposed system was tested through a MATLAB simulation experiment.
\end{abstract}

Keywords: wireless sensor network; mobile communication; cattle tracking; cattle rustling

\section{Introduction}

The Kenyan livestock farming is mainly for food and income generation. However, it also has more significance as a symbol of wealth and status among the pastoralist communities. Therefore, there are two distinct livestock producers in Kenya, the pastoralists and the farmers. The farmers are either in subsistence or commercial cattle production. One of the main challenges facing the pastoralists is cattle rustling. The commercial cattle farmers are also faced with rampant cases of cattle theft. Cattle raiding and cattle rustling have a long history as an aspect of the traditional pastoralist culture. Cross-border cattle rustling violence is common among the Karamojong community of Uganda and the Turkana people of Kenya. In the semi-arid northern Kenya, armed cattle rustling violence is common among the pastoralist communities of Samburu, Turkana, and the Pokot. In November 2012 more than 40 policemen were killed by Turkana cattle rustlers as they sought to recover animals stolen in a violent cattle rustling conflict involving Turkana and Samburu pastoralists [1]. Trying to follow animal tracks without knowing their exact location always puts the lives of the recovery personnel at risk which in most cases has resulted in a high number of military and civilian casualties. Considering the benefits and features of mobile communication, wireless sensor networks, and advancements in animal identification, we propose a framework that provides a secure and convenient way to identify and track stolen animals in areas faced with regular cattle rustling and livestock theft.

Several studies have tried to implement wireless sensor networks in animal tracking, behavior monitoring, and activity control. An implementation of wireless sensor devices to monitor the state of 
the animals and the landscape within a paddock has been implemented by [2], while [3] implemented virtual fences for controlling the movement of cows relative to the fence boundary for automatically herding the cows in the absence of physical fences. The studies in [2,3] concluded that new wireless sensor devices could be developed with different engineering trade-offs to existing devices and meet the needs of particular areas of applications. One usage of wireless sensor networks (WSN) in animal management that has not been explored is the application of wireless sensor networks systems in alleviating cattle rustling. The contribution of this paper is a framework for alleviating cattle rustling through animal identification and tracking in harsh terrain using mobile communication assisted wireless sensor networks. The paper is organized as follows: Section 2 discusses existing work in electronic animal identification and tracking. Section 3 defines the requirements for the proposed conceptual framework system and gives an overview of the proposed framework. Finally, Section 4 gives conclusions and discusses future work.

\section{Animal Identification and Tracking}

The concept of livestock tracking has been around for some time now. Global positioning system (GPS) and wireless technologies have over the years been used for animal tracking using technologies such as the radio frequency identification (RFID) tags [4] and GPS satellite collars [5,6]. Between 1998 and 2001, one million animals were electronically identified using ear tags, the stomach bolus, and the injectable transponders in the European commission through the Identification Electronique des Animaux (IDEA) project [7]. The Ministry of Agriculture in Botswana uses the Livestock Identification Trace-back System (LITS), an RFID based animal identification technology to capture data on individual cattle, and stores this data in a central database [8]. LITS uses rumen boluses with embedded RFID microchips [9] to trace animals with each rumen bolus coded with the owner's name, a personal identification number, the sex of the animal, the hide color of the animal and the location of the animal. This data is then uploaded to an extension officers' computer and stored on a central database in the capital city of Botswana, Gaborone. The rumen bolus is a small plastic or ceramic capsule which rests at the rumen and is inserted down the throat of an animal using a special administration tool.

For the last few years WSN has been used in monitoring the health of dairy cows [10-12]. A multi-year study by the Commonwealth Scientific and Industrial Research Organization (CSIRO), Australia, have been investigating the use of WSN for automated livestock monitoring and control as cattle sensor networks [13]. Most of these cases have used collar mounted sensory devices for implementing WSN in animal management such as the famous applications in the ZebraNet [14] and cattle sensor networks [13-15] or positioned sensors in the Great Duck Island [16]. An experiment was conducted on an implicit routing protocol (IRP) to be used in a cost effective WSN for monitoring the health of dairy cows [17]. The IRP was to mitigate the effects of animal mobility on the network performance which affects real-time data reporting through high packet loss and long delay in WSN. However, all of these experiments were done in constrained environments which are different from tracking cattle in case of cattle rustling or theft in terms of the sensor nodes used, distance travelled by the animals, and animal movements. Several readymade collars and receivers from different manufacturers are available off the shelf, but with the nature of cattle rustling, a mounted device on an animal cannot be used as it can be removed and left on the ground and the animal moved to a different location. It could also be removed and placed by the rustlers in a different location and direction to lure the recovery team away from the rustlers' track. One way to track the animals as they move would be to use the proposed approach to tracking moving targets using video sensors connected to strategically deployed nodes [18]. The main challenge with use of the video sensors is that it requires them to be placed in strategic positions in a presumed path the cattle rustlers would use as they move with the animals. However, in cattle rustling, the direction and the paths taken by the raiders are not well defined nor predefined.

Animal identification is the combination and linking of the identification and registration of an animal individually with a unique identifier [19]. Identification of the animal without tracking 
live animals is counterproductive. A traceability study done in Kenya by [20] indicated that when moving cows from Garisa to Kwale, one of the registered animals was received in Malindi (one of the checkpoints) but did not reach the intended final destination (Kwale) after it strayed. This indicates that even though the animal had the required identification rumen bolus, its location could not be identified. Tracking is the capacity to isolate the current estimated location of an animal using the identifying device. Therefore, there is the need to couple identification with tracking in cattle management. Proper tracking involves real-time identification of the animal's location to enable successful tracking of the animal. It is easy to identify the position of an animal in a WSN as the position of the sensor node, where the animal would be hosted, is known in the sensor network [21].

\section{Proposed Framework}

The proposed cattle tracking and recovery system consists of a rumen sensor module, a WSN control unit, a Worldwide Interoperability for Microwave Access (WiMAX) gateway, WiMAX base stations, and a data center. The rumen sensor module acquires the real-time location data of the animal and sends the data to the wireless control unit. The WSN Control Unit (WCU) module, which is a multi-protocol WSN coordinator, controls the rumen sensor module and transfers the data to the data center for storage using WiMAX connectivity. The Database Management System (DBMS) will be used for storing the extracted data in a database. The system's database is based on TinyDB. TinyDB is a lightweight document oriented database, which facilitates data collection and storage from the sensors using power-efficient in-network processing algorithms. Below is the proposed framework for the livestock identification, tracking and recovery (Figure 1). It is a solution for livestock identification and tracking that meets the requirements of the people and security personnel in charge of livestock protection and recovery and which provides insight into anti-stock theft network deployments.

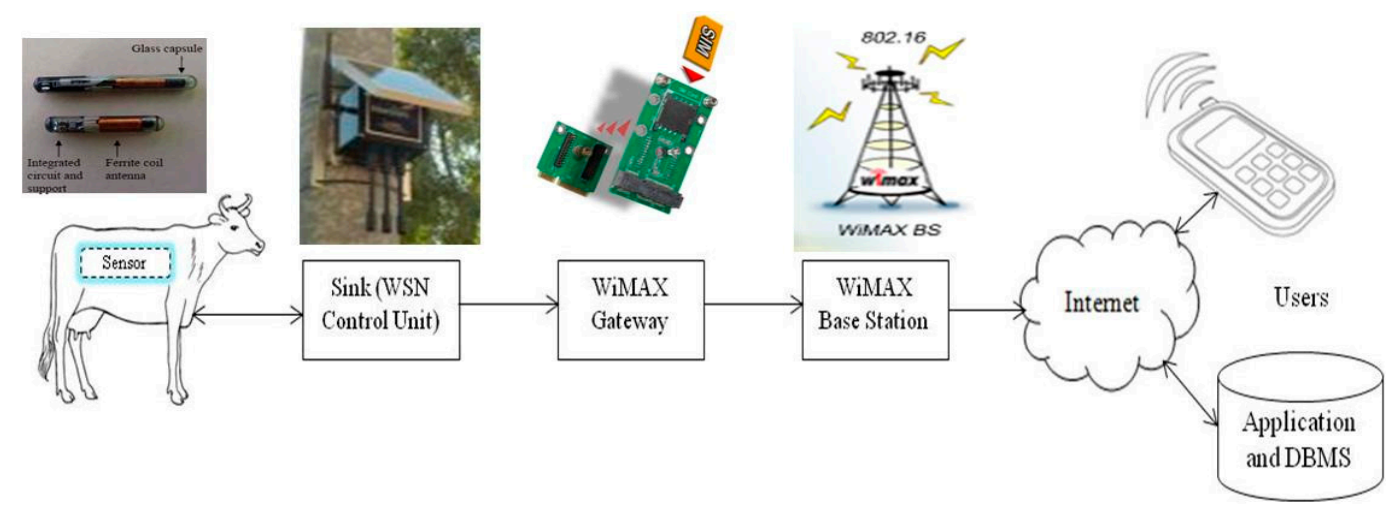

Figure 1. Basic system structural framework.

The system architecture is a three-tiered mobile infrastructure. It has the following key components: The mobile embedded sensor (MES), the WSN control unit, WiMAX gateway, WiMAX base stations, and the application data center. The MES will be inserted in the target animal using the right applicator tool. The source of power for most sensors has always been limited. One suggestion on how to address this has been to use Wireless Power Transfer solution which provides a means for electronic devices to obtain power wirelessly without any physical connectivity to the WSN nodes attached to the animals [22]. The WSN control unit and WiMAX gateway will have an infinite energy source, high computation power, and large storage. The MES does not communicate with its neighbors always and it does not deliver data until contacted by WSN control unit.

\subsection{Mobile Embedded Sensor Node}

In most wireless sensor networks, the sensor nodes operate from fixed locations or in constrained environments, but in cattle tracking the sensor nodes should be moving with the animal hence the 
challenges of mobility and reliable network connectivity. Tracking mobile objects have been studied by [23-25]. A mobile embedded sensor does not control its movement as it is attached or placed in the target. In animal management, it is usually tethered on to the animal [14], placed in the animal rumen [13], or inserted under the animal skin [26]. Finkenzeller [27] indicates that there are four types of transponders that can be affixed to an animal, including collar mounted transponders, ear tags, injectable transponders, and rumen bolus transponders. The collar mounted transponders and ear tags can easily be removed and left on the ground as the cattle thief moves with the animals. There are also concerns with use of injectable transponders. The injectable transponders have been reported to migrate from the site of application [28]. The desired sensor node for this study should have the capability to hold the identification information of the target as well as collect the data relating to the target geographic location. Therefore, we propose use of a bolus transponder as a passive sensor node to locate the animal.

Currently, the rumen bolus is an RFID based transponder for animal identification. Some have thermistors to measure the temperature and are activated to transmit acquisitions after insertion into the animal. One key advantage of using RFID is that it does not require clear line of sight for reading as with the GPS based systems. Other advantages of using RFID include low cost, its small size which means that it can be easily mounted in an animal as an implant, and its long life as it lacks the need for a constant energy supply [29]. However, RFID could be unreliable in its readings, especially in cases when animals move too fast or many of them are present near the reader [29]. RFID is also limited in its maximum reading distance which is just a few meters. These RFID limitations make it unreliable for cattle tracking in case of cattle rustling. Use of virtual fencing or geo-fencing such as in [30,31] is not applicable as herders usually drive animals over long distances during dry season in search of water and grass. We suggest the use of a rumen-based device that could be an improved version of what was used by [32]. Use of a matchbox sized MICA2 $433 \mathrm{MHz}$ motes placed inside standard drug release capsules would be the most appropriate device. Even though [32] concentrates on the internal health statistics of the animals, the motes used were able to communicate over $300 \mathrm{~m}$. This is an indication that if the device is supported with an appropriate localization protocol and a distant estimation algorithm, it could be used to get the current position of the animal. A uniform random deployment will be used when selecting the animals to host the motes. The nodes will not have a priori information about their location and this has to be estimated using a wireless sensor network localization algorithm. Each sensor node will normally operate in sleep mode and will be individually localized by passively receiving wireless control unit messages with minimal inter-node communications.

\subsection{Gateway}

The WSN control unit is a multi-protocol WSN coordinator equipped with a WiMAX gateway device. The gateway is composed of the WSN control unit and the WiMAX gateway. Several manufacturers have tried to create wireless sensor network devices which combine WSN sink coordinator and other wireless communications technologies. Such devices include the Centre Suisse d'Electronique et de Microtechnique (CSEM) WiseGSM (Global System for Mobile Communications) [33] and BeanAir BeanGateway [34] devices which combine WSN with General Packet Radio Service (GPRS). Also studies such as [35-37] utilize GSM/GPRS modem with embedded Transmission Control Protocol and the Internet Protocol (TCP/IP) stack to provide wireless TCP-IP connectivity to WSN for remote monitoring. This study proposes to use Waspmote gateway [38] by libelium which has a dedicated socket for solar panel input, hardware interfaces for a 3G plus GPS module, and a maximum communication range of 7 to $12 \mathrm{~km}$. The Waspmote will integrate all the information received from the sensor nodes and calculate their locations and send the text data to the database using the WiMAX gateway. Multiple gateways should be deployed in the neighboring villages using a square grid in which the number of gateways will be determined a priori when planning the WSN and depending on the village size. The initial deployment should maintain network coverage and connectivity using a minimum number of gateways. The static gateway maintains a 
connection to the server using WiMAX connectivity. The gateways should be fitted with a renewable energy source such as solar power for longer term autonomous operation. The gateways should have a priori information about their coordinates. A schematic diagram of the gateway is shown in Figure 2 .

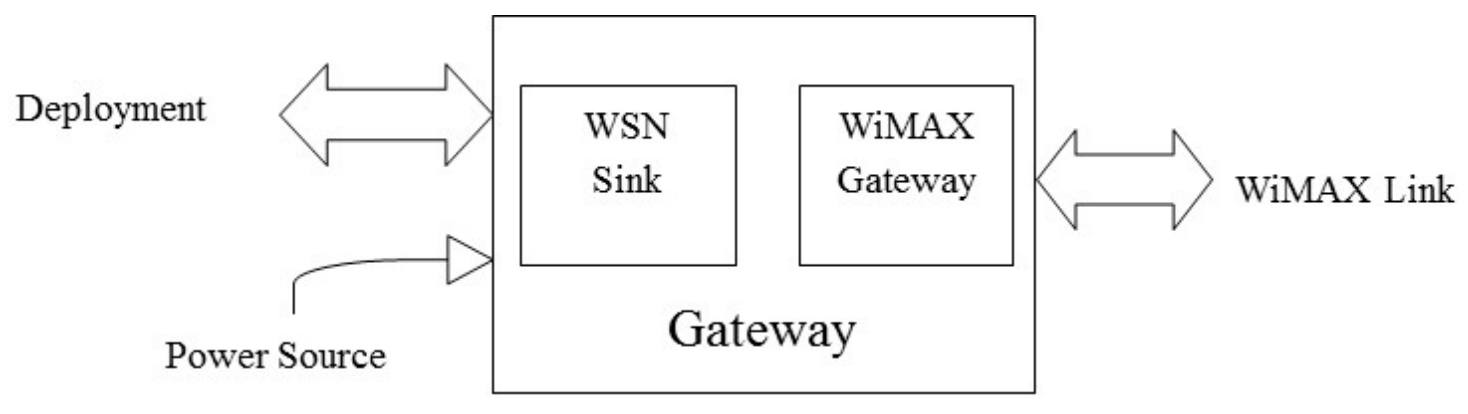

Figure 2. Block schematic of the gateway.

\subsection{System Operations}

The target animals could be in different locations within the village. At the beginning, each gateway propagates its location coordinates to the targets within its reach. We propose that only $10 \%$ of animals will be used as targets in any target environment. The assumption is that the target animals will be evenly distributed in any groupings of the entire animal population. Each target begins its movement at a random start location. It is assumed that the animals use random walk mobility model which puts no constraint on the target velocity or moving direction. The animal moves from its current location by randomly choosing a direction and speed in which to plod within the grazing area. The average animal walking speed is $4.5 \mathrm{~km} / \mathrm{h} \mathrm{[3]} \mathrm{and} \mathrm{there} \mathrm{is} \mathrm{an} \mathrm{indication} \mathrm{that} \mathrm{if} \mathrm{the} \mathrm{animal}$ is being rushed, it could double this speed. The data is transferred from the boluses to the gateways over single hop communication after a given period of time. The gateways store the information and only relay the information to the databases periodically at set intervals from where the users can query the data remotely over the Internet using a computer or mobile telephone. Localization of the target can also be done using multiple gateways being relocated from one location to the other on a mobile platform for real time tracking.

\subsection{Animal Sensor Localization}

Localization is the process of determining the position of an object in space [39]. Sensors are randomly distributed across the entire target area with uniform density. All sensors have the same characteristics including the target identity and radio range. After the sensor node has been deployed, the WSN control unit assists in their localization. The localization algorithm considers direct readings from the WSN control unit to the sensor. An acknowledgment mechanism is adopted whereby the node receiving the packets sends a positive acknowledgment to the sending WSN control unit. The hop count value in the message will be incremented as soon as a node receives the message.

The animal sensor localization is done using distance vector-hop (DV-hop) [40], a distributed hop by hop positioning algorithm. The DV-hop algorithm is a low energy consumption localization scheme, from which many algorithms have been proposed over the last few years [41-44]. Compared to the other range-free localization schemes, the DV-hop algorithm has the capability to handle cases in which a node has less than three neighbor anchors. This makes the DV-hop algorithm more appropriate for use in tracing the animal locations in case of cattle rustling. The DV-hop algorithm localization process has three stages: the localization information exchange phase for obtaining hop counters, average hop distance computation phase for every anchor, and estimated position phase using trilateration or maximum likelihood estimation method $[45,46]$. 
Using the DV-hop localization algorithm an unknown MES calculates its position based on the received WCU locations, the average distance per hop and the hop count from the corresponding WCU using the following three steps [46].

Step 1: The WSN control unit broadcasts a message containing its position and hop count field set to 0 .

Step 2: The anchor receives positions of other anchors as well as the minimum hop counts to other anchors and converts the hop count value into a physical distance.

Step 3: The unknown node location is estimated by the multilateration method using the distance estimations from at least three anchors.

Tracking seeks to identify the position of an object over time [39]. Accurate and energy efficient location estimation is essential for any successful deployment and application of WSN. A received signal strength indicator (RSSI) is used to measure the distance between mobile node and WSN control unit. Each sensor node is within range of at least one WSN control unit and receives its transmission from the WSN control unit.

The following notations are used in the description of the algorithm:

WCU: WSN control unit with GPS

Total number of WCUs in the network $=\left\{w_{1}, w_{2}, \ldots, w_{n}\right\}$

WSID (WSN coordinator number): Unique Waspmote ID (Sink ID)

MES: Animal rumen sensor

Total number of MESs in the network $=\left\{m_{1}, m_{2}, \ldots, m_{\mathrm{z}}\right\}$

MESID (mobile embedded sensor number): Unique animal rumen sensor ID.

HC_Table (Hope Count Table): Maintained by MES and holds data on the minimum number of hops to any three nearest WCU. Fields in this table are WSID, $X_{i}, Y_{i}$, hop ${ }_{i}$.

AS_Table: (Animal sensor table) Maintained by the WCU. It contains information about the coordinates of the localized MES. Fields in this table are AID (Animal number), $X_{i}, Y_{i}$, coordinates of MES.

P_Table (Location or Position table): Maintained by the WCU. It contains information about the node path. Fields in this table are AID, DIST (Distance travelled by the node), VEL (Velocity of the node), $X, Y$ (the node's last known location).

3.4.1. Initialization Phase: To Obtain the Minimum Number of Hops between the MES and Each WCU

Obtaining the Minimum Hop Count

1. Initialize WCU

2. For each WCU

a. Send "Awake" plus "Hello" messages to all MES in the range

For MES set $\mathrm{M}=\left\{m_{1}, m_{2}, m_{3}, \ldots, m_{n}\right\}$

Initialize MES

Set MES status = "active"

The format of WCU "Hello" message is $\left\{\right.$ wsid, $x_{i}, y_{i}$, hop count $\}$

3. MES: Ignore higher hop count values from the same WCU, storing only the minimum hop count to each WCU.

4. MES: Increase the hop counter by 1 and pass the message to the neighbor.

5. Repeat until each MES has received communication from all the WCUs in which it is within their radio ranges and all the MES in the network have the minimal hop count to every WCU.

6. MES: Update HC_Table with $\left(X_{i}, Y_{i}, h o p_{i}\right)$ where $\left(X_{i}, Y_{i},\right)$ is the coordinate of $\mathrm{WCU}_{\mathrm{i}}$ and hop is the minimum number of hops between the MES and the $\mathrm{WCU}_{\mathrm{i}}$. 


\subsubsection{Computation Phase: To Calculate Estimated Distance between MES and WCU}

(a) To calculate the per-hop average distance

1. The WCU uses the minimum hop count values to other WCUs to estimate the average size of one hop within the network using Equation (1).

$$
\text { HopSize }_{i}=\frac{\sum_{j \neq i} \sqrt{\left(x_{i}-x_{j}\right)^{2}+\left(y_{i}-y_{j}\right)^{2}}}{\sum_{j \neq i} h_{i j}}
$$

where $x_{i}, y_{i}$ and $x_{j}, y_{j}$ are the beacon coordinates of WCUs $i$ and $j, h$ is the minimum number of hops between beacon WCUs $i$ and $j$.

2. WCU broadcasts its average hop size to the network using controlled flooding to the entire network.

3. Each MES receives the hop sizes from the nearest WCUs and saves the first received message and transmits it to the neighbors.

(b) To calculate the physical distance between MES and WCU

MES computes its estimated physical distance to the WCUs using the received hop size using Equation (2).

$$
d_{m w}=\text { HopSize }_{i} \times h o p_{m w}
$$

where $h o p_{m w}$ is the minimum hop-count between the unknown $\mathrm{MES}_{\mathrm{m}}$ and the $\mathrm{WCU}_{\mathrm{w}}$, HopSize $_{i}$ is the average hop size that the $\mathrm{MES}_{\mathrm{m}}$ obtained from the nearest $\mathrm{WCU}_{\mathrm{w}}$.

\subsubsection{Localization Phase: Coordinate Calculation}

Using the estimated physical distance in computation phase the MES calculates its coordinates using the maximum likelihood estimate of trilateration using the algorithm derived in [31] as Equation (3).

$$
\left\{\begin{array}{c}
\left(x-x_{1}\right)^{2}+\left(y-y_{1}\right)^{2}=d_{1}^{2} \\
\left(x-x_{2}\right)^{2}+\left(y-y_{2}\right)^{2}=d_{1}^{2} \\
\cdots \\
\left(x-x_{i}\right)^{2}+\left(y-y_{i}\right)^{2}=d_{1}^{2}
\end{array}\right.
$$

Equation (3) is then transformed to into a linear equation in [31] as Equation (4) which is used in computing the coordinates of the unknown node.

$$
X=\left(A^{T} A\right)^{-1} A^{T} B
$$

\subsection{Localization Accuracy}

Localization accuracy is determined by how close the estimated position of a node is close to the actual coordinates of the unknown node. An algorithm is deemed to have a good localization accuracy if the node's estimated position is very close to its actual coordinates. Most range free localization algorithms are prone to location accuracy errors. The error between the actual and estimated MES coordinates of a node can be calculated using Equation (5) as the localization error.

$$
\text { Location Error }=\sqrt{\left(x_{2}-x_{1}\right)^{2}+\left(y_{2}-y_{1}\right)^{2}}
$$

where $\left(x_{1}, y_{1}\right)$ are the actual MES coordinates, and $\left(x_{2}, y_{2}\right)$ are the estimated MES coordinates. 
The positioning efficiency of the algorithm is the average location error of all the nodes relative to the radio range of the nodes and can be calculated using Equation (6).

$$
\text { Average Location Error }=\frac{\text { error }_{1}}{R}
$$

where error 1 represents the average value of localization error and $R$ represents communication radius. The smaller the accuracy value, the more accurate it the algorithm is.

\section{Simulation and Results}

To evaluate and validate the performance of the animal sensor localization, thorough simulation tests were done. MATLAB Simulink software was used as the tool to build the simulation environment. The simulation parameters are given in Table 1 . The simulation results are given as average localization error of 100 experiments.

Table 1. Default simulation configurations.

\begin{tabular}{cc}
\hline Parameters & Default Values \\
\hline Network Area & $1000 \times 1000 \mathrm{~m}$ \\
Simulation Time & $180 \mathrm{~s}$ \\
Mobile target velocity $(\lambda)$ & $1 \sim 10 \mathrm{~m} / 60 \mathrm{~s}$ \\
Range Nodes Propagation Range & $\mathrm{R}=300 \mathrm{~m}$ \\
Number of Nodes & $200(10 \%$ of 2000 animals $)$ \\
Placement of Nodes & Random \\
Number of Gateways & 11 (Vary gateways between 5 and 15$)$ \\
Placement of Gateways & Deterministic (but random in this case) \\
Mobility Model Used & Random Walk Mobility Model \\
\hline
\end{tabular}

In a given population of 2000 animals, $10 \%$ of the animals would be hosting the animal bolus sensors. In Figure 3, 200 sensor nodes are placed randomly in a deployment area. The deployment area of the wireless sensor network is considered as $1000 \times 1000 \mathrm{~m}$ with 200 randomly deployed sensor nodes as depicted in Figure 3.

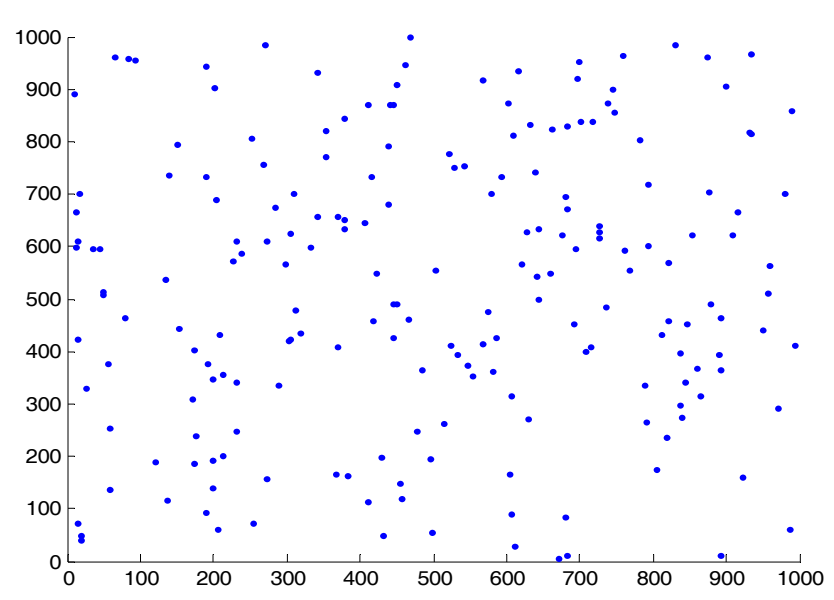

Figure 3. Node Distribution (Random) at a given time.

Figure 4, shows the graphical representation of node placement and the identification of unknown node positions with respect to the anchor nodes. One hundred and eight nine sensor nodes are deployed with 11 anchors, the estimated distance values away from 3 different anchors are sufficient for an unknown node to calculate its location. If the network is continuously working, the animals which are within the range of the network could be tracked by identifying their current position. 


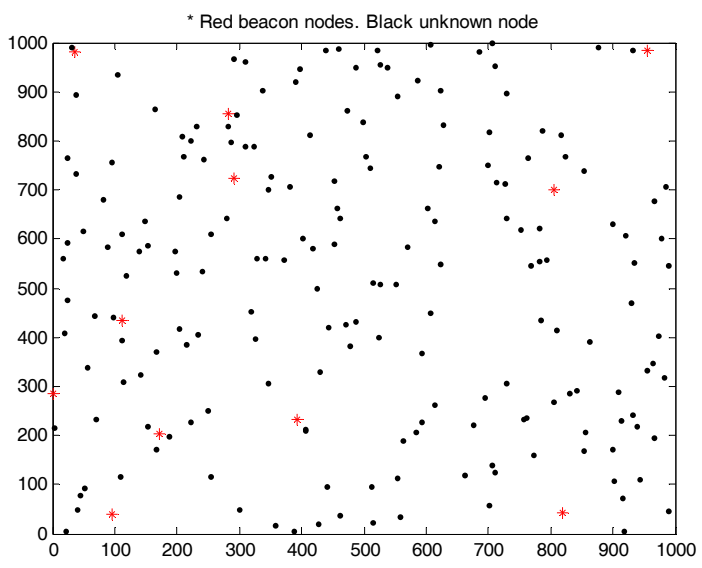

Figure 4. Node distribution with Beacons, where * represents the anchor node and $\mathbf{~ r e p r e s e n t s ~ t h e ~}$ unknown node.

The distribution of the nodes is shown in Figure 4. The anchors and nodes are randomly distributed with some placement error. There are 200 sensor nodes and 11 gateways deployed. The placement of the anchor nodes has a high impact on how effective the localization and identification of the current animal location as the nodes are in motion most of the time. The localization errors, Figure 5, are represented by the ratio of the Euclidean distances between estimated coordinates and actual nodes coordinates, scaled to the communication radius $R$.

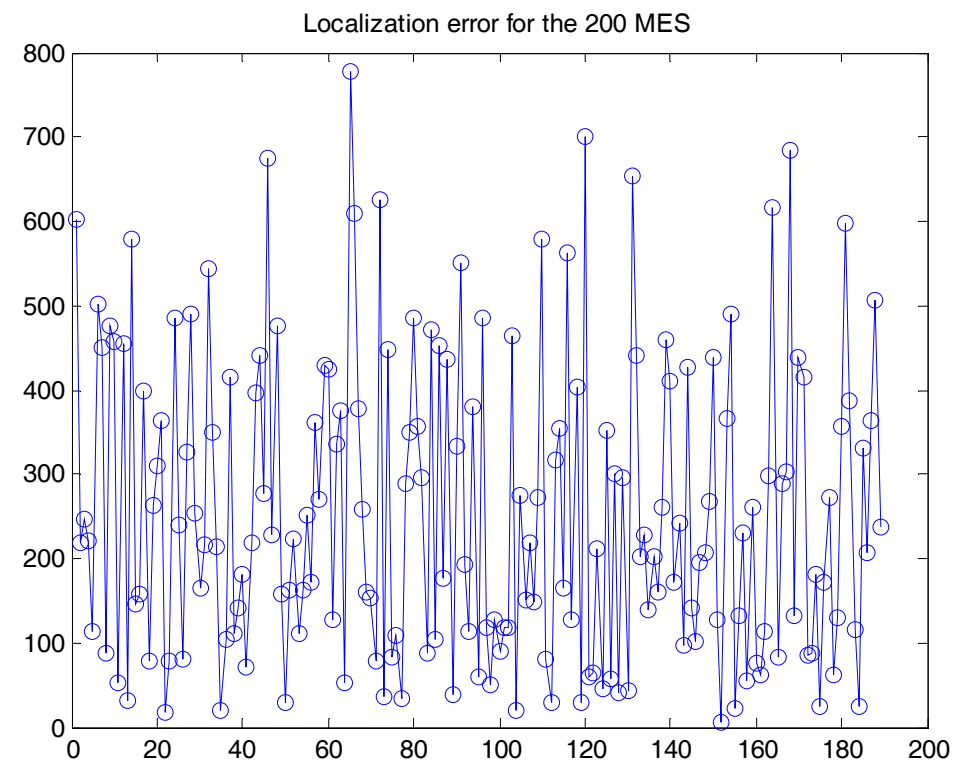

Figure 5. Localization error for the 200 unknown nodes.

Localization error as a function of the radio transmission range is given for the random scenarios in Figure 5. Considering a ranging error of $25 \mathrm{~m}$, the nodes are localized to a very good accuracy. The average error is also found to be $8.8 \mathrm{~m}$. the position estimates obtained are exceedingly good. Every additional unknown node will behave like a beacon assisting other unknown nodes. The average error in the position estimate of unknown nodes in Figure 5 is also reduced to $7.51 \mathrm{~m}$.

It is indicated in [47] that the quantity of beacons and their placement reduce the localization error. The more proportionate placement of beacons lowers the localization error while increasing the number of beacons also reduces the localization error [47]. To reduce the localization errors, 
the proposed system could further be enhanced using the Grey Wolf Optimization algorithm which to provides better results in finding the location of unknown nodes [48].

\section{Conclusions and Future Work}

Like many other studies which have tried to integrate WSNs with mobile communication technologies such as RFID [49,50] studies, this paper has proposed a WSN based multi-technology framework. WSNs have been widely used for wildlife monitoring and tracking of different [51]. This paper describes our ongoing work in use of WSN to facilitate quick and safe recovery of stolen animals hence defeating the purpose of cattle rustling. There are plans to implement this conceptual framework in a test environment and eventually extend the experiment to the field. The test environment could also be extended to have some of the animals host special MES and to collect data using WiMAX or GPRS/GSM to the Internet. A module for data collection using UAV that visits some static nodes in the grazing fields as applied by [52] could also be used to enhance the proposed system. The systems could also have the UAV gather the sensed data in the animal sensors so as to reduce the battery power consumption and bandwidth usage as proposed by [53]. The boluses could also be used in monitoring the health of the host animal, hence offering multiple benefits to the host animals. The proposed framework would be a great improvement on the current preferred methods including ear tags, hot-iron branding, and ear notching [54]. We also aim to develop extension to the proposed framework.

Acknowledgments: This work was facilitated by the Federation of Indian Chambers of Commerce \& Industry (FICCI) and the Indian Institute of Information Technology, Allahabad (IIIT-A), through an International Postdoc Fellowship Program.

Conflicts of Interest: The author declares no conflict of interest.

\section{References}

1. Momanyi, B. Kenyan Cops Killed in Ambush Hit Shocking 42. Available online: http:/ / www.capitalfm.co. ke/news/2012/11/kenyan-cops-killed-in-ambush-hit-shocking-42/ (accessed on 21 October 2013).

2. Sikka, P.; Corke, P.; Overs, L. Wireless sensor devices for animal tracking and control. In Proceedings of the 29th Annual IEEE International Conference on Local Computer Networks, Tampa, FL, USA, 16-18 November 2004.

3. Butler, Z.; Corke, P.; Peterson, R.; Rus, D. Virtual fences for controlling cows. In Proceedings of the 2004 IEEE International Conference on Robotics and Automation, New Orleans, LA, USA, 26 April-1 May 2004; pp. 4429-4436.

4. Frost, A.R.; Schofield, C.P.; Beaulah, S.A.; Mottram, T.T.; Lines, J.A.; Wathes, C.M. A review of livestock monitoring and the need for integrated systems. Comput. Electron. Agric. 1997, 17, 139-159. [CrossRef]

5. Tomkins, N.; Filmer, M. GPS tracking to boost sustainability. Farming Ahead 2007, 185, 68-71.

6. Raizman, E.A.; Rasmussen, H.B.; King, L.E.; Ihwagi, F.W.; Douglas-Hamilton, I. Feasibility study on the spatial and temporal movement of Samburu's cattle and wildlife in Kenya using GPS radio-tracking, remote sensing and GIS. Prev. Vet. Med. 2013, 111, 76-80. [CrossRef] [PubMed]

7. Ribo, O.; Korn, C.; Meloni, U.; Cropper, M.; de Winne, P.; Cuypers, M. IDEA: A large-scale project on electronic identification of livestock. Rev. Sci. Tech. OIE 2001, 20, 426-436. [CrossRef]

8. Moreki, J.C.; Ndubo, N.S.; Ditshupo, T.; Ntesang, J.B. Cattle Identification and Traceability in Botswana. J. Anim. Sci. Adv. 2012, 2, 925-933.

9. Bowling, M.B.; Pendell, D.L.; Morris, D.L.; Yoon, Y.; Katoh, K.; Belk, K.E.; Smith, G.C. Review: Identification and Traceability of Cattle in Selected Countries Outside of North America. Prof. Anim. Sci. 2008, 24, 287-294. [CrossRef]

10. Erden, H.; Kaya, İ.A.; Çamaşircioğlu, E. Livestock monitoring system. In Proceedings of the Fourth International Conference on Agro-Geoinformatics, Istanbul, Turkey, 20-24 July 2015; pp. 73-76.

11. Bhavsar, A.R.; Shah, D.J.; Arolkar, H.A. Distributed data storage model for cattle health monitoring using WSN. Adv. Comput. Sci. Int. J. 2013, 2, 19-24. 
12. Sasloglou, K.; Glover, A.; Kwong, K.H.; Andonovic, I. Wireless sensor network for animal monitoring using both antenna and base-station diversity. In Proceedings of the IEEE ICCS 08, Guangzhou, China, 19-21 November 2008.

13. Wark, T.; Corke, P.; Sikka, P.; Klingbeil, L.; Guo, Y.; Crossman, C.; Valencia, P.; Swain, D.; Bishop-Hurley, G. Transforming agriculture through pervasive wireless sensor networks. IEEE Pervasive Comput. 2007, 6, 50-57. [CrossRef]

14. Zhang, P.; Sadler, C.M.; Lyon, S.A.; Martonosi, M. Hardware design experiences in ZebraNet. In Proceedings of the 2nd International Conference on Embedded Networked Sensor Systems, Baltimore, MD, USA, 3-5 November 2004; pp. 227-238.

15. Handcock, R.N.; Swain, D.L.; Bishop-Hurley, G.J.; O’Neill, C.J. Monitoring animal behaviour and environmental interactions using wireless sensor networks, GPS collars and satellite remote sensing. Sensors 2009, 9, 3586-3603. [CrossRef] [PubMed]

16. Mainwaring, A.; Polastre, J.; Szewczyk, R.; Culler, D.; Anderson, J. Wireless sensor networks for habitat monitoring. In Proceedings of the 1st ACM International Workshop on Wireless Sensor Networks and Applications, Atlanta, GA, USA, 28 September 2002; pp. 88-97.

17. Wu, T.T.; Goo, S.K.; Kwong, K.H.; Michie, C.; Andonovic, I. Wireless sensor network for cattle monitoring system. GIL Jahrestagung 2009, 142, 173-176.

18. Sanchez, A.J.G.; Sanchez, F.G.; Losilla, F.; Kulakowski, P.; Haro, J.G.; Rodríguez, A.; Bao, J.V.L.; Palomares, F. Wireless sensor network deployment for monitoring Wildlife Passages. Sensors 2010, 10, 7236-7262. [CrossRef] [PubMed]

19. World Organisation for Animal Health (OIE). Terrestrial Animal Health Code; World Organisation for Animal Health: Paris, France, 2011.

20. Matete, G.O.; Maingi, N.; Muchemi, G.; Ogara, W.; Gathuma, J.M. Design and development of an electronic identification and traceability system for cattle under pastoral production systems: A case for Kenya. Livest. Res. Rural Dev. 2010, 22, 1-17.

21. Ghumare, S.S.; Labade, R.P.; Gagare, S.R. Rare Wild Animal Tracking in the Forest area with Wireless Sensor Network in Network Simulator-2. Int. J. Comput. Appl. 2016, 133. [CrossRef]

22. Beng, L.T.; Kia, P.B.; Meng, L.N.; Cheng, P.N. Field testing of IoT devices for livestock monitoring using Wireless Sensor Network, near field communication and Wireless Power Transfer. In Proceedings of the IEEE Conference on Technologies for Sustainability, Airport Marriott, Phoenix, AZ, USA, 9-11 October 2016.

23. Kusy, B.; Ledeczi, A.; Koutsoukos, X. Tracking mobile nodes using rf doppler shifts. In Proceedings of the 5th International Conference On Embedded Networked Sensor Systems, Sydney, Australia, 6-9 November 2007; pp. 29-42.

24. Brooks, R.R.; Griffin, C.; Friedlander, D.S. Self-organized distributed sensor network entity tracking. Int. J. High Perform. Comput. Appl. 2002, 16, 207-219. [CrossRef]

25. Li, X.R.; Jilkov, V.P. A survey of maneuvering target tracking: Approximation techniques for nonlinear filtering. In Proceedings of the 2004 SPIE Conference on Signal and Data Processing of Small Targets, San Diego, CA, USA, 13-15 April 2004; pp. 537-550.

26. Caja, G.; Ndez-Jover, M.H.; Conill, C.; Garı́n, D.; Alabern, X.; Farriol, B.; Ghirardi, J. Use of ear tags and injectable transponders for the identification and traceability of pigs from birth to the end of the slaughter line. J. Anim. Health 2005, 83, 2215-2224. [CrossRef]

27. Finkenzeller, K. Rfid Handbook, 3rd ed.; John Wiley \& Sons, Ltd.: Great Britain, UK, 2010.

28. Hogewerf, P.H.; Ipema, A.H.; Binnendijk, G.P.; Lambooij, E.; Schuiling, H.J. Using injectable transponders for sheep identification. In Proceedings of the 7th European Conference on Precision Agriculture, Wageningen, The Netherlands, 6-8 July 2009.

29. Radenkovic, M.; Wietrzyk, B. Mobile Ad Hoc Networking approach to detecting and querying events related to farm animals. In Proceedings of the IEEE International Conference on Networking and Services, Silicon Valley, CA, USA, 16-18 July 2006; pp. 109-115.

30. Bishop-Hurley, G.J.; Swain, D.L.; Anderson, D.M.; Sikka, P.; Crossman, C.; Corke, P. Virtual fencing applications: Implementing and testing an automated cattle control system. Comput. Electron. Agric. 2017, 56, 14-22. [CrossRef]

31. Filmer, M. Virtual fences set to transform farming. Farming Ahead 2007, 190, 70-71. 
32. Mayer, K.; Taylor, K.; Ellis, K. Cattle health monitoring using wireless sensor networks. In Proceedings of the 2nd IASTED International Conference on Communication and Computer Networks, Cambridge, MA, USA, 8-10 November 2004; pp. 27-33.

33. CSEM Scientific and Technical Report 2009. Available online: http:/ /www.csem.ch/docs/Show.aspx/12633/ docname/CSEM-STR09-Page\%2025.pdf (accessed on 24 April 2017).

34. A Multi-Protocol Wireless Sensor Networks Coordinator. Available online: http://www.beanair.com/ beangateway (accessed on 24 April 2017).

35. Sveda, M.; Benes, P.; Vrba, R.; Zezulka, F. Handbook of Sensor Networks: Compact Wireless and Wired Sensing Systems; Taylor \& Francis: Abingdon, UK, 2005; pp. 10-24.

36. Bencini, L.; Palma, D.D.; Collodi, G.; Manes, A.; Manes, G. Wireless Sensor networks for on-field agricultural management process. In Wireless Sensor Networks: Application-Centric Design; Merrett, G.V., Tan, Y.K., Eds.; In Tech Open Access Publisher: Rijeka, Croatia, 2010; pp. 1-18.

37. Jain, J.N.; Agrawala, A.K. Open Systems Interconnection: Its Architecture and Protocols; Elsevier: Amsterdam, The Netherlands, 1990.

38. Waspmote Technical Guide. Available online: http://www.libelium.com/downloads/documentation/ waspmote_technical_guide.pdf (accessed on 24 April 2017).

39. Manley, E.D.; Nahas, H.A.; Deogun, J.S. Localization and tracking in sensor systems. In Proceedings of the IEEE International Conference on Sensor Networks, Ubiquitous, and Trustworthy Computing, Newport Beach, CA, USA, 5-7 June 2006; Volume 2, pp. 237-242.

40. Niculescu, D.; Nath, B. Ad Hoc Positioning System (APS). In Proceedings of the IEEE GLOBECOM, San Antonio, TX, USA, 25-29 November 2001; Volume 5, pp. 2926-2931.

41. Chen, H.; Sezaki, K.; Deng, P.; So, H.C. An improved DV-Hop localization algorithm with reduced node location error for wireless sensor networks. IEICE Trans. Fundam. Electron. Commun. Comput. Sci. 2008, 91, 2232-2236. [CrossRef]

42. Boukerche, A.; Horacio, A.B.F.; Nakamura, E.F.; Antonio, A.F. DV-Loc: A scalable localization protocal using voronoi diagrams for wireless sensor networks. IEEE Wirel. Commun. 2009, 16, 50-55. [CrossRef]

43. Kumar, S.; Lobiyal, D.K. An advanced DV-hop localization algorithm for wireless sensor networks. Wirel. Pers. Commun. 2013, 71, 1365-1385. [CrossRef]

44. Hu, Y.; Li, X.M. An improvement of DV-hop localization algorithm for wireless sensor networks. Telecommun. Syst. 2013, 53, 13-18. [CrossRef]

45. Heng, F. Algorithm of wireless sensor network node based on the vehicle localization. In Proceedings of the 3rd International Conference on Mechatronics, Robotics and Automation (ICMRA), Shenzhen, China, 20-21 April 2015.

46. Zhu, J.; Lv, C.; Tao, Z. An improved localization scheme based on DV-Hop for large-scale wireless sensor networks. J. Commun. 2016, 11, 1057-1065.

47. Yin, M.; Shu, J.; Liu, L.; Zhang, H. The influence of beacon on DV-hop in wireless sensor networks. In Proceedings of the Fifth International Conference on Grid and Cooperative Computing Workshops, Changsha, China, 21-23 October 2006.

48. Rajakumar, R.; Amudhavel, J.; Dhavachelvan, P.; Vengattaraman, T. GWO-LPWSN: Grey wolf optimization algorithm for node localization problem in wireless sensor networks. J. Comput. Netw. Commun. 2017. [CrossRef]

49. Zhang, L.; Wang, Z. Integration of RFID into wireless sensor networks: Architectures opportunities and challenging problems. In Proceedings of the IEEE Fifth International Conference on Grid and Cooperative Computing Workshops, Changsha, China, 21-23 October 2006; pp. 463-469.

50. Pereira, D.P.; Dias, W.R.A.; Braga, M.L.; Barreto, R.S.; Figueiredo, C.M.S.; Brilhante, V. Model to integration of RFID into wireless sensor network for tracking and monitoring animals. In Proceedings of the Eleventh IEEE International Conference on Computational Science and Engineering, Nagoya, Japan, 23-25 August 2008; pp. 125-131.

51. Ou, C.H.; He, W.L. Path planning algorithm for mobile anchor-based localization in wireless sensor networks. IEEE Sens. J. 2013, 13, 466-475. [CrossRef]

52. Xu, J.; Solmaz, G.; Rahmatizadeh, R.; Turgut, D.; Bölöni, L. Animal monitoring with unmanned aerial vehicle-aided wireless sensor networks. In Proceedings of the 40th IEEE Conference on Local Computer Networks (LCN), Clearwater Beach, FL, USA, 26-29 October 2015; pp. 334-341. 
53. Kang, J.; Larkin, H. Application of an emergency alarm system for physiological sensors utilizing smart devices. Technologies 2017, 5, 26. [CrossRef]

54. Bett, B.; Mutua, F.; Kihara, A.; Rogena, J.; Ngwili, N.; Muunda, E.; Wabacha, J. Piloting Livestock Identification and Traceability Systems in Pastoral Production Systems in Eastern Africa; Preliminary Report; ILRI: Nairobi, Kenya, 2015. 